\title{
Evaluating the Usability of the Leap Motion Controller
}

\author{
Dominic King \\ Department of Computing \\ University of Central Lancashire \\ Preston, Lancashire, PR1 2HE \\ dking5@uclan.ac.uk
}

\author{
Matthew Horton \\ Department of Computing \\ University of Central Lancashire \\ Preston, Lancashire, PR1 2HE \\ MPLHorton@uclan.ac.uk
}

\author{
Dev Raj Lamichhane \\ Department of Computing \\ University of Central Lancashire \\ Preston, Lancashire, PR1 2HE \\ drlamichhane@uclan.ac.uk
}

\begin{abstract}
The Leap Motion Controller (LMC) allows for hand gesture movements to be tracked in order to conduct various tasks in a desktop orientated environment. With the use of gesture recognition and natural user interfaces, it allows for fluent and natural communication, via the use of hand movements. Various topics have been discussed in relation to LMC, many looking at various communication theories and the robustness of the device. This research examined the LMC functions and identified key usability issues related to the application "Form and Function 3D", providing an adequate solution for usability.
\end{abstract}

Gesture Recognition; Leap Motion Controller; User Interaction; Natural User Interaction;

\section{INTRODUCTION}

Gesture recognition technology is a relatively new and diverse area of study, with a variety of virtual environments currently available. When observing hand gestures within an $\mathrm{HCl}$ context it has been described as being an active field of research, providing a vast area to delve into when studying a user's actions via hand movements (Badi \& Hussein, 2014). Despite the surge of virtual reality and motion detection systems, it is important to consider key user experiences and how users generally interact with modern day systems.

This study aims to identify key usability issues when navigating with a Leap Motion Controller (LMC) using a built-in application called "Form \& Function 3D". Leap Motion allows users to navigate on a desktop PC using mainly hand movements to control user interfaces (Fisseler et al. 2013). The controller pays close attention to palm direction, fingertip position and relevant hand movement (Lu et al. 2016).

Gesture Motion is a distinct and vast topic, with various different research interests at heart. For the usability of a system to be monitored and for error rates to be formed, user centred observational studies can be considered a suitable option. Usability can be described as an expression used to describe computer systems which are relatively easy to use by untrained users (Issa \& Turk, 2012).

In order to investigate this, a user study of the Form and Function 3D application was conducted, where participants used their hands to complete a set of tasks using the Leap Motion Controller. The main focus of this paper relates highly on the gained insights of the user's experience, aiming to identify the usability of the Leap Motion Controller and the errors which may surface.

\section{BACKGROUND}

This section addresses the key topic areas that are related to the study which are Natural User Interfaces, Leap Motion and Form and Function 3D application.

\subsection{Natural User Interfaces and Leap Motion}

Natural User Interface (NUI) is terminology used in $\mathrm{HCl}$ where the surface is invisible and is typically based on human natural elements (Sousa et al. 2015). NUI based consumer devices have become much more widely available however, such technologies have long been proven the most challenging in computer science (Lecklider, 2011).

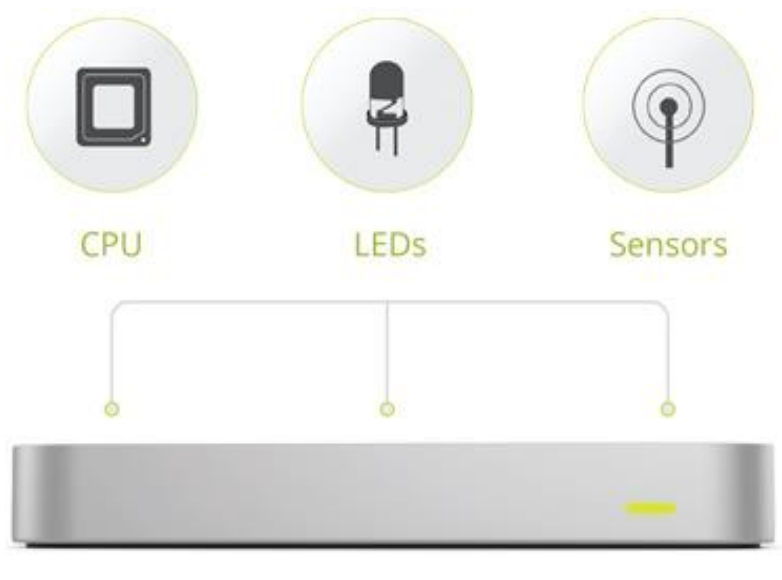

Figure 1: Leap Motion sensing hardware (Shilov, 2013)

Due to the surge in natural user interfaces (NUIs), they build upon human-to-human interaction models, and in order to define a consistent interaction between a device and the user, research had to be carried out effectively, for the data to be valuable (Wang et al. 2015). Leap Motion introduces a new gesture and position tracking system with 
submillimetre accuracy as seen in Figure 1 (Weichert et al. 2013). The LMC has most of its tracking software directly above the hardware rather than in front of the device, therefore allowing users to interact primarily by hovering their hands above the sensors.

Leap Motion permits a natural user interface, allowing users to use gestures to interact with a virtual environment. According to research, the LMC provides an error rate of around $7.8 \%$ opposed to general mouse devices, highlighting the limitations it may have when conducting tasks (Bachmann et al. 2014). This research will explore the user interaction with the Leap Motion detector, identifying key flaws the device may have, and the struggles users encounter.

\subsection{Gesture Recognition}

With the use of gesture recognition technologies, it allows the recognition of instinctive actions to occur, detecting hand movements in real time. In this instance, the research looks at gesture based user interfaces, measuring the movement of the human hand. Gesture recognition brings frequent challenges that can affect the accuracy and usefulness of the device (Rama \& Mohod, 2014). The hand is an important part of the human body and in the fields of human computer interaction, many studies have been carried out in order to recognise hand gesture constraints (Du et al. 2017).

The majority of research on the Leap Motion Controller falls heavily on the robustness of gesture capabilities and the fundamental problems associated with it (Weichert, 2013). With the goal of Human Computer Interaction being to improve communication between users, dynamic hand gestures are important for recognising key hand movements such as waving, in order for users to communicate effectively (Haria et al. 2017).

\subsection{Form and Function 3D Application}

Form and Function 3D is a downloadable education tool on the LMC which allows users to explore different animal anatomy (Brendan, 2017). Many features are available within the application allowing individuals to identify structures of real life 3D organs, blood flow simulations, participate in quiz's and various other tasks. This application was deemed the most useful and worthwhile application to use for a user study due to the diversity, allowing users to conduct particular tasks, whilst taking note of key usability issues the application presents and any issues the Leap Motion Controller causes.

\section{USER STUDY}

In order to gain an insight on the gesture functionality of the Leap Motion Controller, suitable tasks were designed so that can they can be carried out with a user centred observation by participants using the Form and Function 3D application, to identify key usability issues and errors. The Leap Motion will track gesture recognition from the user's hand movements allowing for an understanding on a user's ability to communicate via gesture recognition. The research questions the paper wishes to answer is, how useful are gesture orientated applications when it comes to enticing users to view and understand data naturally.

Risk factors were identified before conducting the experiment assessing potential risks that may go wrong. The two most important factors consisted of loss of computer system due to power failure and distress to the participant from gesture activities. All of these had countermeasures in place, providing a number of backup computers and regular breaks to relieve the users stress. Ethical issues are present when conducting data collection, and following Walton's (2016) research, various steps are taken to countermeasure any issues.

Users were provided with informative consent ensuring they continually understand the information they are provided with and the chance to leave the study at any given time.

\subsection{Method}

The purpose of this study was to capture individuals performing a vast variety of tasks using a Leap Motion Controller, providing an insight into the usability and error rates related to the device. There are various methods to evaluate a product's usability such as heuristic evaluation, cognitive walkthroughs and user studies, however, with heuristics focusing more on theoretical models, user studies became the more evident to use, due to exploratory learning techniques (Bligärd \& Osvalder, 2013).

With this user centred approach, user performance can be visually monitored identifying how they interact to particular tasks, taking into consideration key design flaws. The apparatus consisted of a Leap Motion Controller and a MacBook Pro, set out in an isolated environment to minimise any conditional variation between participants and reduce the chance of distraction.

\subsection{Participants}

This study consisted of four participants who, through a prior experience questionnaire, were deemed to have a good level of experience with technology, two of which had prior experience interacting with NUI technologies, opposed to the 
other two, who had very little knowledge of the technology. This divide in participants was chosen to help identify key usability issues and solutions for both novice and experienced users of the technology.

\subsection{Procedure}

The experiment was carried out in a controlled environment with one participant using the device at a time on separate occasions to reduce the chance of any learning effects, affecting the study. As the individual arrived, an instruction sheet was provided along with a consent form to be read and signed before being able to participate in the research. These sheets informed the user of what the experiment entailed, how the data will be stored, and any terms and conditions needed to sign beforehand.

Once the instructions were confirmed and consent forms signed, a task sheet was provided to the individual with further instructions. The experiment consisted of a user centred observation of the Form and Function 3D application, carrying out a number of tasks which relate to the usability of the application and the LMC. There were 5 tasks in total, all of which were timed at 5 minute intervals allowing 5 minutes to complete each task. The experiment took an approximately 45 minutes to complete for each participant, due to regular break intervals after each task.

All of the given tasks were achievable in respect to the software, and the skills are all set up and active on the Leap Motion prior to the experiment being carried out. Once a task was completed, time was given for the participant to rest due to the length of time they were required to use their hands. The overall experiment was informal and situated in a fun environment to avoid any stressful situation however, throughout the experiment numerous participants did become agitated and frustrated with the Leap Motions sensor due to the lag between their gestures and responses from the system.

\section{FINDINGS AND DISCUSSION}

When the participants began the tasks, observation forms were used to capture key usability issues that the user may face and which area of the interface it affects, along with severity. Each participant was observed using data capture forms, identifying key problems related to navigation, content, interaction and the presentation of the system, along with any additional comments the user may have faced.

This final information was stored and merged into a table highlighting all of the key problems faced, and merging them into smaller, related incidents to identify the frequency of occurrence (see table 1).
Throughout the study it became evident that several of the participants began to become agitated and required help with some of the tasks they faced. Many of the tasks from all participants did not get completed altogether, identifying key usability issues. Participant $A$ and $D$, were participants that had prior experience with NUI technologies, opposed to participants $B$ and $C$ who had very little knowledge of the technology. The results highlighted how participants $B$ and $C$ did not manage to complete 7 out of 10 tasks they faced in total, highlighting the difficulty of the system.

Participants $A$ and $D$ did not manage to complete 3 of the tasks out of the 10 they both faced, highlighting that even though the level of difficulty is reduced, they still encountered troublesome moments. This identifies a divide within the participants highlighting those with prior experience struggled less than those without. However, not all participants managed to finish every task completely, identifying areas for improvement within the application.

Table 1: Merged table of the problems encountered

\begin{tabular}{|l|l|}
\hline \multicolumn{1}{|c|}{ Problems } & Severity \\
\hline Unable to swipe correctly & 4 \\
\hline Unable to locate "explore" on the cat organ & 3 \\
\hline $\begin{array}{l}\text { Kept pointing at the screen due to thinking } \\
\text { the circular button was a helper not a button }\end{array}$ & 4 \\
\hline Unaware of how to rotate the organ & 3 \\
\hline No idea of how to inspect the organ & 3 \\
\hline $\begin{array}{l}\text { Unaware as to what the 'help' feature would } \\
\text { look like }\end{array}$ & 2 \\
\hline $\begin{array}{l}\text { Became agitated with the presentation of the } \\
\text { content }\end{array}$ & 3 \\
\hline Transparency feature not clear enough & 2 \\
\hline Thought the reset button was the exit button & 4 \\
\hline Lagged pointer & 3 \\
\hline $\begin{array}{l}\text { Organ span uncontrollably with the hand } \\
\text { movement }\end{array}$ & 3 \\
\hline $\begin{array}{l}\text { Buttons are not clear as to how to work them } \\
\text { correctly }\end{array}$ & 2 \\
\hline
\end{tabular}

Numerous issues with the Form and Function 3D application became evident, with a substantial list of concerns and usability issues identified in the study (see table 1). The problems shown in table 2, highlight the most frequently occurring problems that occurred for more than one participant as well as the severity level associated with it.

Table 2: Issues participants found the most problematic

\begin{tabular}{|l|c|c|c|c|}
\hline \multicolumn{1}{|c|}{ Problems encountered } & \multicolumn{4}{|c|}{ Participants } \\
\cline { 2 - 5 } & $A$ & $B$ & $C$ & $D$ \\
\hline Unable to swipe correctly & - & - & & \\
\hline $\begin{array}{l}\text { Unable to locate "explore" on the organ } \\
\text { page }\end{array}$ & & & & - \\
\hline
\end{tabular}




\begin{tabular}{|l|l|l|l|l|}
\hline Unaware on how to rotate the organ & & & $\checkmark$ & \\
\hline Transparency feature not clear enough & & & & \\
\hline Organ span uncontrollably & & & & \\
\hline
\end{tabular}

With these three main usability issues identified it highlights the key areas in which the application may lack in terms of user experience. In order to identify the most problematic tasks to finalise a solution, all participants were asked which of the top five most identified usability issues they found the most problematic. Table 2 identifies, that two participants believe swiping is the major flaw, along with two other participants stating that when unable to locate options and rotate information, it became problematic, identifying the three main factors for improvement.

From the list of results, a vast majority of usability issues are evident, highlighting that some participants looked endlessly at the screen with no idea as to what the interaction possibilities were. The pointer lagged significantly which can be related to the performance of the LMC itself or in relation to the sensors within the device. Users became agitated with the layout of the content and the way it was unresponsive in certain aspects and this particular design can be classed as bad practice.

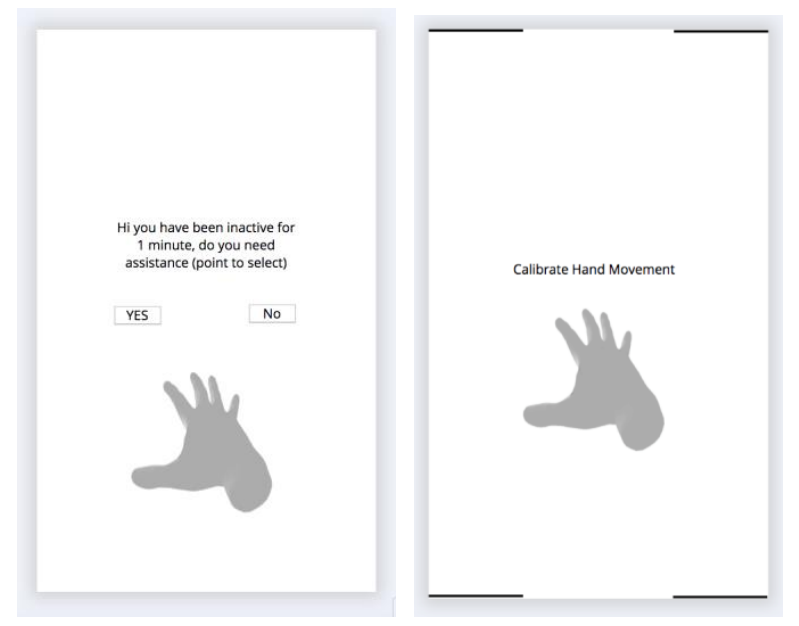

Figure 2: Form and Function 3D Application Solution

A solution in order for the LMC and Form and Function 3D Application to developer a friendlier and user orientated experience taking into consideration key design principles, they may inject new means of transferring information. Information is difficult to digest, hand gestures are lagging in motion, simple gestures are not easy to navigate and no feedback was provided at all to the user. Feedback elements may provide a new means of communication and help assist VR users in order to accomplish particular tasks with little helper to make the content more readable and user friendly (see Figure 2 ).
The main solution would be to inject a notification element within the overall design structure to enable users to receive constant feedback as to what they are doing and if they may need any advice or guidance. This could be implemented in various areas of the application to assist the user with any key usability issues they are facing. Another solution could be to calibrate the hand movements with the LMC, as seen in Figure 2, allowing users to point to all corners of the device screen to calibrate their hand movement. This may reduce lagging and assist with the major flaw which was the swipe not working correctly.

The results highlight significant usability issues and limitations for the future of the application and the LMC itself in terms of accuracy with its sensors. In relation to the research question, the data is not understood naturally, with various interventions throughout the tasks, therefore identifying that users are less enticed and more agitated when in these situations. Future research may delve into routes that involve more participants to conduct the research and may use several different applications that are more user friendly. This research can assist and influence the next phase of the design for Form and Function 3D providing key evidence their application has an array of usability issues and causes a frustration in user activity.

\section{CONCLUSION}

Whilst this research has been relatively successful in locating key usability issues with the LMC, further research can be applied in order to expand the understanding of the user experience, and the error rates that arise from such actions. This research primarily collected data from a small sample size and a larger size may identify any further usability issues.

The research identified key usability factors and focused mainly on three of the main causes, providing adequate solutions to combat these issues. The data highlights how users struggled to control gestures, locate specific information and unaware how to communicate with particular content. These flaws can be ideally combatted with well formatted content, calibrated hand movements and integrated notifications.

\section{REFERENCES}

Bachmann, D., Weichert, F. and Rinkenauer, G., 2014. Evaluation of the Leap Motion Controller as a New Contact-Free Pointing Device. Sensors, 15(1), pp. 214-233. 
Badi, H. and Hussein, S., 2014. Hand posture and gesture recognition technology. Neural Computing and Applications, 25(3), pp. 871-878.

Bligård, L. and Osvalder, A., 2013. Enhanced Cognitive Walkthrough: Development of the Cognitive Walkthrough Method to Better Predict, Identify, and Present Usability Problems. Advances in Human-Computer Interaction, 2013.

Bremin, S., Bång, M. and Jönsson, A., 2011. Rapid evaluation of TV interaction devices using a Cognitive Walkthrough method.

Brendan, P., 06/30, 2017-last update, Form and Function 3D [Homepage of Leap Motion], [Online]. Available: https://gallery.leapmotion.com/form-andfunction-3d/ [01/20, 2018].

Du, Y., Liu, S., Feng, L., Chen, M. and Wu, J., 2017. Hand Gesture Recognition with Leap Motion.

Fisseler, D., Bartholomäus R, Bachmann, D. and Weichert, F., 2013. Analysis of the Accuracy and Robustness of the Leap Motion Controller. Sensors, 13(5), pp. 6380-6393.

Haria, A., Subramanian, A., Asokkumar, N., Poddar, S. and Nayak, J.S., 2017. Hand Gesture Recognition for Human Computer Interaction. Procedia Computer Science, 115, pp. 367-374.

Issa, T. and Turk, A., 2012. Applying Usability and $\mathrm{HCl}$ Principles in Developing Marketing Websites.

Lecklider, T., 2011. Your scope needs a natural user interface. EE: Evaluation Engineering, 50(1), pp. 1222.

Li, T., Wang, D., Peng, C., Yu, C. and Zhang, Y., 2018. Speed-accuracy trade-off of fingertip force control with visual/audio/haptic feedback. International Journal of Human Computer Studies, 110, pp. 33-44.

Lu, W., Tong, Z. and Chu, J., 2016. Dynamic Hand Gesture Recognition with Leap Motion Controller. Signal Processing Letters, IEEE, 23(9), pp. 1188-1192.

Rama, D.B. and Mohod, P.S., 2014. Survey on Hand Gesture Recognition Approaches. International Journal of Computer Science and Information Technologies, 5(2), pp. 2050-2052.
Shilov, A., 02/27, 2013-last update, Motion Sensing PC and Mac Controller Slips to May, But Promises Wide Availability [Homepage of xbitlabs], [Online]. Available: http://www.xbitlabs.com/news/multimedia/ display/20130227233301_Leap Motion Controller Set to Ship in May Available for Global Pre Or ders_Now.html $[10 / 05,2016]$.

Sousa, L., Monteiro, J., P.J.S. Cardoso and J.M.F. Rodrigues, 2015. Natural user interface based on gestures recognition using Leap Motion sensor. Dos Algarves: A Multidisciplinary e-Journal, 26(1), pp. 107-121.

Walton, I., 2016. Ethical Research. Midwifery Matters, (151), pp. 18-20.

Wang, X., Bernardos, A.M., Besada, J.A., Metola, E. and Casar, J.R., 2015. A gesture-based method for natural interaction in smart spaces. Journal of Ambient Intelligence \& Smart Environments, 7(4), pp. 535-562.

Weichert F., 2013. Analysis of the accuracy and robustness of the leap motion controller.

Weichert, F., Bachmann, D., Rudak, B. and Fisseler, D., 2013. Analysis of the accuracy and robustness of the leap motion controller. Sensors, 13(5), pp. 63806393. 